\title{
Coupling Pumped Hydro Energy Storage with Unit Commitment
}

\author{
K. Bruninx, Student Member, IEEE, Y. Dvorkin, Student Member, IEEE, E. Delarue, Member, IEEE, \\ H. Pandžić, Member, IEEE, W. D'haeseleer and D. S. Kirschen, Fellow, IEEE
}

\begin{abstract}
Renewable electricity generation provides affordable and emission-free electricity, but also introduces additional complexity in the day-ahead planning procedure. To address the stochastic nature of renewable generation, system operators must schedule enough controllable generation to have the flexibility required to compensate unavoidable real-time mismatches between the production and consumption of electricity. This flexibility must be scheduled ahead of real-time and comes at a cost, which should be minimized without compromising the operational reliability of the system. Energy storage facilities, such as pumped hydro energy storage (PHES), can respond quickly to mismatches between demand and generation. Hydraulic constraints on the operation of PHES must be taken into account in the day-ahead scheduling problem, which is typically not done in deterministic models. Stochastic optimization enhances the procurement of flexibility, but requires more computational resources than conventional deterministic optimization. This paper proposes a deterministic and an interval unit commitment formulation for the co-optimization of controllable generation and PHES, including a representation of the hydraulic constraints of the PHES. The proposed unit commitment (UC) models are tested against a stochastic UC formulation on a model of the Belgian power system to compare the resulting operational cost, reliability and computational requirements. The cost-effective regulating capabilities offered by the PHES yield significant operational cost reductions in both models, while the increase in calculation times is limited.
\end{abstract}

Index Terms-Flexibility, unit commitment, pumped hydro energy storage, stochastic optimization, interval optimization, wind energy.

\section{NOMENCLATURE}

\section{A. Sets and Indices}

$I$

$J$

$R$

$S$

$s^{\mathrm{F}}$

$S^{\mathrm{R}}$

$s_{\mathrm{e}}^{\mathrm{R}+}$

$s_{\mathrm{O}}^{\mathrm{R}+}$

$s_{\mathrm{e}-}^{\mathrm{R}-}$

Set of power plants, index $i$.

Set of time steps, index $j$.

Set of PHES units, index $r$.

Set of wind power output scenarios, index $s$.

Index of the central wind power forecast scenario.

Set of wind power ramping scenarios, index $s^{R}$. Index of the maximum upward wind power forecast error ramp scenario on even time steps. Index of the maximum upward wind power forecast error ramp scenario on odd time steps. Index of the maximum downward wind power forecast error ramp scenario on even time steps.

K. Bruninx, E. Delarue and W. D'haeseleer are with the University of Leuven (KU Leuven) Energy Institute, TME Branch (Energy Conversion), B-3001 Leuven, Belgium.

H. Pandžić is with the Faculty of Electrical Engineering and Computing University of Zagreb, Zagreb HR-10000, Croatia.

Y. Dvorkin and D. S. Kirschen are with the Department of Electrical Engineering, University of Washington, Seattle, WA 98195-2500, USA.

Corresponding author: William D'haeseleer, tel: $+3216 / 322510$, fax: $+3216 / 322985$, e-mail: william.dhaeseleer@mech.kuleuven.be.

$s_{\mathrm{O}}^{\mathrm{R}-}$
B. Decision
co $_{2} c_{i, j, s}$
$e_{r, j, s}$
$f c_{i, j, s}$
$g_{i, j, s}$
$g_{r, j, s}^{\mathrm{PHS}, \mathrm{P}}$
$g_{r, j, s}^{\mathrm{PHS}, \mathrm{T}}$
$L L_{j}$
$r_{i, j}^{+}$
$r_{i, j}^{-}$
$r_{r, j}^{\mathrm{PHS}, \mathrm{P},+}$
$r_{r, j}^{\mathrm{PHS}, \mathrm{P},-}$
$r_{r, j}^{\mathrm{PHS}, \mathrm{T},+}$
$r_{r, j}^{\mathrm{PHS}, \mathrm{T},-}$
$r c_{i, j, s}$
$s c_{i, j}$
$\Delta^{+} g_{r, j, s}^{\mathrm{PHS}, \mathrm{P}}$

Index of the maximum downward wind power forecast error ramp scenario on odd time steps.

\section{B. Decision Variables}

$\mathrm{CO}_{2}$-emission cost of power plant $i$ at time step $j$ under scenario $s, €$.

Energy storage level of PHES $r$ at time step $j$ under scenario $s$, MWh.

Fuel cost of power plant $i$ at time step $j$ under scenario $s, €$

Output of power plant $i$ at time step $j$ under scenario $s$, MW.

Consumption of PHES $r$ in the pumping mode at time step $j$ under scenario $s$, MW.

Output of PHES $r$ in the turbining mode at time step $j$ under scenario $s$, MW.

Lost load at time step $j$, MW.

Upward reserve provided by power plant $i$ at time step $j$, MW.

Downward reserve provided by power plant $i$ at time step $j$, MW.

Upward reserve provided by PHES $r$ in the pumping mode at time step $j$, MW.

Downward reserve provided by PHES $r$ in the pumping mode at time step $j$, MW.

Upward reserve provided by PHES $r$ in the turbining mode at time step $j$, MW.

Downward reserve provided by PHES $r$ in the turbining mode at time step $j$, MW.

Ramping cost of power plant $i$ at time step $j$ under scenario $s$, €.

Start-up cost of power plant $i$ at time step $j$, €. Auxiliary variable to enable feasibility of downward reserve provision in the pumping mode of PHES $r$ at time step $j$ under scenario $s$, MW.

$\Delta^{-} g_{r, j, s}^{\text {PHS,P }}$ Auxiliary variable to enable feasibility of upward reserve provision in the pumping mode of PHES $r$ at time step $j$ under scenario $s$, MW.

$\Delta^{+} g_{r, j, s}^{\mathrm{PHS}, \mathrm{T}}$ Auxiliary variable to enable feasibility of downward reserve provision in the turbining mode of PHES $r$ at time step $j$ under scenario $s$, MW.

$\Delta^{-} g_{r, j, s}^{\mathrm{PHS}, \mathrm{T}} \quad$ Auxiliary variable to enable feasibility of upward reserve provision in the turbining mode of PHES $r$ at time step $j$ under scenario $s$, MW.

$\alpha_{r, j, s} \quad$ Binary variable indicating the turbining and pumping status of the PHES. If $\alpha_{r, j, s}=0$ $\left(\alpha_{r, j, s}=1\right)$, PHES $r$ is in the pumping (turbining) mode at time step $j$ under scenario $s$.

Wind spillage at time step $j$ under scenario $s$, MW. 


\section{Parameters}

$D_{j} \quad$ Demand at time step $j$, MW.

$D_{j}^{+} \quad$ Upward reserve requirement at time step $j, \mathrm{MW}$.

$D_{j}^{-} \quad$ Downward reserve requirement at time step $j$, MW.

$E_{r}^{\mathrm{MAX}} \quad$ Maximum energy storage level of PHES $r$, MWh.

$E_{r}^{\mathrm{MIN}} \quad$ Minimum energy storage level of PHES $r$, MWh.

$N^{\mathrm{MC}} \quad$ Number of scenarios used for Monte Carlo evaluation of the day-ahead schedule.

$P_{r}^{\mathrm{MAX}} \quad$ Maximum power capacity of PHES $r$, MW.

$P_{s} \quad$ Probability of scenario $s$.

TP Length of the time step $j, \mathrm{~h}$.

VOLL Value of lost load, €/MWh.

$W_{j, s} \quad$ Wind power output under scenario $s$ at time step j, MW.

$\epsilon_{r} \quad$ Round-trip efficiency of PHES $r$.

\section{INTRODUCTION}

$\mathbf{T}$ HE simplest way to help system operators reliably handle the increased variability and uncertainty caused by the integration of wind power generation is to increase the amount of reserves [1]. Under conventional day-ahead planning procedures, this is implemented by exogenously increasing the reserve requirement in the Deterministic Unit Commitment (DUC) formulation [2], [3]. While this approach is straightforward, it results in a significant increase in the operational cost because generators and energy storage resources are dispatched in a less economic manner [4], [5] in order to meet this more stringent reserve constraint. Although the cost performance of these reserve policies can be improved by means of parametric [6] or non-parametric [7] statistical analyses, the exogenous nature of these requirements inhibits the co-optimization of the reserve amount with the Unit Commitment (UC) decisions. On the other hand, stochastic [8], interval [9], and robust [10] formulations of the UC problem endogenously account for the stochastic nature of wind power generation. Typically, these optimization techniques yield more cost-efficient UC schedules than the deterministic technique because of their more flexible approach to the scheduling of flexible resources.

Papavasiliou et al. [8] demonstrated that the Stochastic UC (SUC) formulation achieves a lower operational cost than a DUC model with the $(3+5)$ reserve policy [11] on the CAISO test system. Similarly, simulations on the ISO New England system have shown that the Robust UC (RUC) is more effective than a DUC [10], if the budget of uncertainty is chosen appropriately. Although both the SUC and RUC formulations are more cost efficient than the DUC, these formulations differ in their computational and cost performance [12]. The SUC model applied to a real-life power system can take tens of hours to be solved, even with a large duality gap and a small number of scenarios [13]. However, Dvorkin et al. [14] also reveal that lowering the duality gap and increasing the number of scenarios does not necessarily improve the accuracy of the SUC approach, leading to a less economic schedule. This can be explained by the dependence of the SUC on the quality of the scenario generation and reduction techniques used to produce a representative set of scenarios [14], [15]. A common flaw of these techniques is that wind power generation is assumed to follow a probability distribution which does not precisely fit empirical data [6], [7], [16] or scenario reduction techniques that fail to identify critical scenarios [15]. However, if one succeeds in capturing the underlying stochasticity in a sufficiently small set of scenarios, resulting in a tractable SUC, this approach yields the optimal decision under uncertainty. The RUC model can be solved faster than the SUC because, unlike the SUC, it models potential realizations of wind power generation as an interval around the central forecast, and thus avoids assumptions regarding individual scenarios [17]. Since the RUC hedges the system against any realization within a given uncertainty set but does not account for the probability of its occurrence, it may produce overly conservative schedules. Therefore, the so-called budget of uncertainty is a crucial mechanism to achieve a good trade-off between the operational cost and robustness of the RUC solution [18]. To the best of the authors' knowledge, there is no systematic way to optimize the value of the budget of uncertainty a priori. This lack of transparency may impede the application of the RUC model in real-life power systems. The conservatism of the RUC can be partially alleviated by constructing uncertainty sets that take into account the temporal and spatial correlations of wind power generation and making non-polyhedral (e.g., ellipsoidal) assumptions on the uncertainty set [17], [19]. However, these uncertainty sets may worsen the computational performance of the RUC model [20].

Wang et al. proposed an Interval Unit Commitment (IUC) formulation [9] that can be viewed as an alternative to the SUC and RUC models. In the IUC model, the set of scenarios used in a SUC is reduced to three distinct scenarios: the central forecast, an upper limit scenario and a lower limit scenario. The IUC is thus less computationally demanding than the SUC because it considers fewer scenarios [21], [22]. However, the SUC generally produces more cost efficient schedules [21]. Like the RUC, the IUC hedges against any realization within the interval around the central forecast defined by the upper and lower limit scenario, thus providing the same level of reliability. However, the RUC model is more computationally demanding due to the "max-min" structure of its subproblem [10].

The cost performance of a UC formulation can also be improved by using additional sources of flexibility, such as energy storage (ES) [23]. However, potential investments in ES must be carefully weighed against their prospective operational efficiency gains [22], [24]. While battery ES and other prospective ES technologies remain expensive [24], system operators have gained significant experience in operating Pumped Hydro ES (PHES), which is by far the most widespread ES technology and is available in many power systems worldwide [25]. Kalantari and Galiana [26] demonstrated that existing PHES can be used to provide critical flexibility to accommodate intermittent wind power generation. Additionally, PHES can be used for improving operational reliability when dealing with intermittent wind power generation [27]. A PHES can also significantly increase the profits of a generating company in a market environment [28]. The common thread 
of the studies in [26]-[28] is that they employ a deterministic framework, which does not account for the possible activation of the regulation services offered by the PHES. The PHES may not be able to deliver the scheduled regulating services, resulting in wind energy spillage or load shedding. Alternatively, stochastic programming techniques can be used to assess the participation of PHES in regulating services ([23], [29] and references therein). As the PHES is dispatched in each scenario modeled in the UC formulation, the hydraulic constraints are satisfied in these scenarios. One must however check that these constraints remain satisfied during dispatch. For example, Jiang et al. [29] propose a RUC with PHES and focus on the computational aspects of this model. Pozo et al. [23] study a SUC including a generic, ideal storage. The SUC yields cost-effective UC and PHES schedules, but is computationally intensive and the solution quality depends on the quality of the scenarios. To the best of the authors' knowledge, no DUC or IUC model has been published that aims to account for the hydraulic constraints of a PHES, while allowing PHES to offer regulating services in order to approximate the solution of a SUC at a significantly lower computational cost.

This work makes the following contributions:

1) We formulate DUC and IUC models that co-optimize the UC and PHES decisions, while taking into account the hydraulic limitations of the PHES. These formulations mirror actual day-ahead system operational practices.

2) Using these DUC and IUC models, we compare different operation strategies of PHES against a benchmark obtained from a SUC. We analyze their impact on the UC decisions and the utilization of the available wind power.

\section{Methodology}

The objective of all UC formulations is to obtain the leastcost schedule that allows meeting the demand for electrical energy subject to given operational constraints and uncertain wind power production. However, these models differ in the way they account for this uncertainty.

The DUC formulation [2] uses probabilistic reserve constraints to estimate potential real-time wind power forecast errors [6]. These constraints represent the upper and lower bounds on the wind power forecast errors for a given probability level. Alternatively, the Improved Interval Unit Commitment (IIUC) accounts for wind uncertainty by modeling the upper and lower bound of the wind power forecast error, i.e. the range of possible wind realizations, and inter-hour ramping scenarios. A feasible dispatch, i.e. a dispatch that causes no load shedding, is enforced for each scenario within a prescribed range of uncertainty [30]. We will consider an IIUC with modified inter-hour ramping scenarios [30], [31]. In the SUC model, the uncertainty is represented directly in the UC model via a larger set of scenarios, including their probability of occurrence. The reserve calculation is internalized in the model. The solution of the SUC will provide a lower bound on the attainable operational cost, given the uncertainty presented by the imperfect wind power forecasts.
Below, we present a stylized version of the UC formulation and only consider spinning reserves, which allows us to focus on the PHES modeling. We start from a SUC, as it is the most general and most direct formulation of a UC model considering uncertainty. The DUC and IIUC formulations can be seen as simplifications of the SUC. The full description of the SUC, considering spinning and non-spinning reserves, can be found in [32], while the DUC and IIUC formulations are given in [2] and [30], respectively.

In all the UC models implemented in this work the network constraints are omitted. This assumption may lead to transmission congestion, especially under high wind penetration levels [33]. However, the Belgian power system that provides the basis for our simulations has enough (internal) transmission capacity to make the effect of congestion essentially negligible [34]. From a market perspective, the Belgian day-ahead market is cleared as one zonal market (with a single price). Transmission constraints are checked in a second stage by the TSO, with potential redispatching to alleviate congestion [34]. Currently, this redispatching affects $0.08 \%$ of the yearly electricity production and increases the annual operating cost by approximately $0.3 \%$ (2.9 million EUR per year) [34]. Since the impact of transmission congestion on the operating cost is very marginal, omitting these constraints does not significantly affect the conclusions of this case study. The omission of transmission constraints significantly reduces the computational burden of the UC models. For example, Papavasiliou et al. [13] report that a transmission-constrained SUC model for a relatively small system with 375 transmission lines requires from several to tens of hours to achieve a reasonably small duality gap, even for a relatively small number of scenarios.

The resulting UC schedules have been tested using MonteCarlo dispatch simulations. The scenarios for wind power forecast errors were generated as discussed in [15], based on a statistical description of the forecast error in [6].

\section{A. Reserves and PHES in SUC}

The total operational cost of generating the demanded electrical energy over the considered time horizon includes the startup costs $\left(s_{i, j}\right)$, the fuel costs $\left(f c_{i, j, s}\right)$, the $\mathrm{CO}_{2}$ emission costs $\left(\mathrm{CO}_{2} c_{i, j, s}\right)$, the ramping costs $\left(r c_{i, j, s}\right)$ and the cost associated with load shedding $\left(L L_{j} \cdot V O L L\right)$.

$$
\begin{aligned}
& \min \sum_{i} \sum_{j}\left[s c_{i, j}+\sum_{s} P_{s} \cdot\left(f c_{i, j, s}+c o_{2} c_{i, j, s}+r c_{i, j, s}\right)\right] \\
& \quad+\sum_{j} \sum_{s} P_{s} \cdot T P \cdot L L_{j} \cdot V O L L
\end{aligned}
$$

In objective function (1), $s c_{i, j}$ is the startup cost for power plant $i$ (set $I$ ) in time step $j$ (set $J$ ), $P_{s}$ the probability of scenario $s$ (set $S$ ) and $T P \cdot L L_{j}$ the volume of lost load $L L_{j}$, valued at $V O L L$ (the value of lost load). TP is the time period, here 15 minutes or 0.25 hours. The UC schedule is independent of the wind power scenarios, resulting in scenarioindependent start-up costs. The output of the scheduled power plants differs per scenario, resulting in different fuel, $\mathrm{CO}_{2}$ emission and ramping costs. This optimization is subject to a number of constraints. First, the demand and supply in each 
scenario must be balanced:

$$
\begin{aligned}
\forall j, \forall s: D_{j}-L L_{j, s}= & \sum_{i} g_{i, j, s}+W_{j, s}-\chi_{j, s} \\
& +\sum_{r}\left(g_{r, j, s}^{\mathrm{PHS}, \mathrm{T}}-g_{r, j, s}^{\mathrm{PHS}, \mathrm{P}}\right)
\end{aligned}
$$

The demand $\left(D_{j}\right)$ must be met by conventional generation $\left(g_{i, j, s}\right)$, wind power $\left(W_{j, s}\right)$, which can be curtailed $\left(\chi_{j, s}\right)$ and the net output of the PHES (index $r$, set $R$ ) in the system $\left(g_{r, j, s}^{\mathrm{PHS}, \mathrm{T}}-g_{r, j, s}^{\mathrm{PHS}, \mathrm{P}}\right) . g_{r, j, s}^{\mathrm{PHS}, \mathrm{T}}$ is the output of the PHES in the turbining mode, $g_{r, j, s}^{\mathrm{PHS}, \mathrm{P}}$ represents the consumption in the pumping mode. Load shedding or wind power curtailment may occur if it reduces the expected operational cost (e.g. by avoiding overly conservative and expensive schedules to meet the load in extreme, and thus unlikely scenarios) or if it allows meeting other operational constraints (e.g. ramping constraints that would be violated by extreme ramps in the wind power output), [31], [32]. Second, the conventional power plants are subjected to several technical constraints, such as minimum and maximum loading levels, ramping constraints and minimum up and down times. These constraints differ per fuel and technology. Finally, the hydraulic constraints of a PHES system $r$ are included as follows via its energy content $e_{r, j, s}$ at each time step $j$ in each scenario $s$ :

$$
\begin{aligned}
\forall r, \forall j, \forall s: e_{r, j, s}= & T P \cdot\left(g_{r, j, s}^{\mathrm{PHS}, \mathrm{P}} \cdot \sqrt{\epsilon_{r}}-\frac{g_{r, j, s}^{\mathrm{PHS}, \mathrm{T}}}{\sqrt{\epsilon_{r}}}\right) \\
& +e_{r, j-1, s}
\end{aligned}
$$

In this equation, $\epsilon_{r}$ is the round trip efficiency of the PHES. The energy content is constrained to a minimum and maximum level:

$\forall r, \forall j: E_{r}^{\mathrm{MIN}} \leq e_{r, j, s} \leq E_{r}^{\mathrm{MAX}}$

The output of the PHES should be positive and is constrained to the capacity of the PHES:

$\forall r, \forall j: 0 \leq g_{r, j, s}^{\mathrm{PHS}, \mathrm{T}} \leq P_{r}^{\mathrm{MAX}} \cdot \alpha_{r, j, s}$

$\forall r, \forall j: 0 \leq g_{r, j, s}^{\mathrm{PHS}, \mathrm{P}} \leq P_{r}^{\mathrm{MAX}} \cdot\left(1-\alpha_{r, j, s}\right)$

where $\alpha_{r, j, s}$ is a binary variable that prevents simultaneous pumping and turbining at time interval $j$. If $\alpha_{r, j, s}=0$, then Eq. (5) yields $g_{r, j, s}^{\mathrm{PHS}, \mathrm{T}}=0$ and, thus, only the pumping mode is feasible. On the other hand, if $\alpha_{r, j, s}=1$, then Eq. (6) yields that $g_{r, j, s}^{\mathrm{PHS}, \mathrm{P}}=0$ and, thus, only the turbining mode is feasible. In the SUC model, we consider a set of scenarios for the wind power production, each with its own probability of occurrence. The PHES is dispatched in each scenario, ensuring the enforcement of the hydraulic constraints. Equations (3) - (6) are sufficient to model the PHES. By definition, the PHES will participate in the (implicit) reserve requirements, here represented via a set of scenarios.

\section{B. Reserves and PHES in DUC}

In the DUC formulation, only one scenario is considered, i.e. the forecasted wind power production $\left(s^{\mathrm{F}}\right)$. The probability of this scenario is thus 1 . Reserve constraints are enforced to ensure real-time mismatches between demand and supply can be compensated. Sufficient upward $\left(D_{j}^{+}\right)$and downward $\left(D_{j}^{-}\right)$ reserves must be available:

$$
\begin{aligned}
\forall j: D_{j}^{+}= & \sum_{i} r_{i, j}^{+}+\chi_{j} \\
& +\sum_{r}\left(r_{r, j}^{\mathrm{PHS}, \mathrm{P},+}+\sum r_{r, j}^{\mathrm{PHS}, \mathrm{T},+}\right) \\
\forall j \mid \chi_{j}=0: D_{j}^{-}= & \sum_{i} r_{i, j}^{-} \\
& +\sum_{r}\left(r_{r, j}^{\mathrm{PHS}, \mathrm{P},-}+\sum r_{r, j}^{\mathrm{PHS}, \mathrm{T},-}\right)
\end{aligned}
$$

Since the DUC considers only one scenario, index $s$ has been dropped. The reserves provided by conventional power plants $\left(r_{i, j}^{+}\right.$and $\left.r_{i, j}^{-}\right)$are restricted by the capacity and ramping rate of a power plant. For the reserves provided by the PHES, we introduce four new variables:

- $r_{r, j}^{\mathrm{PHS}, \mathrm{P},+}$ : upward reserves provided by the PHES by reducing the pumping power;

- $r_{r, j}^{\mathrm{PHS}, \mathrm{P},-}$ : downward reserves provided by the PHES by increasing the pumping power;

- $r_{r, j}^{\mathrm{PHS}, \mathrm{T},+}$ : upward reserves provided by the PHES by increasing the turbining power;

- $r_{r, j}^{\mathrm{PHS}, \mathrm{T},-}$ : downward reserves provided by the PHES by decreasing the turbining power.

These PHES reserves are non-negative and constrained by the capacity of the PHES and the planned output of the PHES:

$$
\begin{aligned}
& \forall r, \forall j: r_{r, j}^{\mathrm{PHS}, \mathrm{P},+} \leq g_{r, j}^{\mathrm{PHS}, \mathrm{P}} \\
& \forall r, \forall j: r_{r, j}^{\mathrm{PHS}, \mathrm{P},-}+g_{r, j}^{\mathrm{PHS}, \mathrm{P}} \leq P_{r}^{\mathrm{MAX}} \\
& \forall r, \forall j: r_{r, j}^{\mathrm{PHS}, \mathrm{T},-} \leq g_{r, j}^{\mathrm{PHS}, \mathrm{T}} \\
& \forall r, \forall j: r_{r, j}^{\mathrm{PHS}, \mathrm{T},+}+g_{r, j}^{\mathrm{PHS}, \mathrm{T}} \leq P_{r}^{\mathrm{MAX}} \\
& \forall r, \forall j: r_{r, j}^{\mathrm{PHS}, \mathrm{P},+}, r_{r, j}^{\mathrm{PHS}, \mathrm{P},-}, r_{r, j}^{\mathrm{PHS}, \mathrm{T},+}, r_{r, j}^{\mathrm{PHS}, \mathrm{T},-} \geq 0
\end{aligned}
$$

However, as we will illustrate below, this formulation is insufficient to allow PHES to offer regulating services in the DUC formulation. Indeed, when scheduling PHES reserves, one should ensure that sufficient energy is stored in the upper basin of the PHES (upward reserves) or that one can store the absorbed energy in the upper basin of the PHES (downward reserves). The limits on the energy content of the PHES should be respected at each time step, in the worst-case scenario (activation of all reserves in one direction):

$$
\begin{aligned}
& \forall r, \forall j: e_{j, r}-T P \cdot \sum_{j^{*}=1}^{j}\left(r_{r, j^{*}}^{\mathrm{PHS}, \mathrm{P},+} \cdot \sqrt{\epsilon_{r}}+\frac{r_{r, j^{*}}^{\mathrm{PHS}, \mathrm{T},+}}{\sqrt{\epsilon_{r}}}\right) \\
& \geq E_{r}^{\mathrm{MIN}} \\
& \forall r, \forall j: e_{j, r}+T P \cdot \sum_{j^{*}=1}^{j}\left(r_{r, j^{*}}^{\mathrm{PHS}, \mathrm{P},-} \cdot \sqrt{\epsilon_{r}}+\frac{r_{r, j^{*}}^{\mathrm{PHS}, \mathrm{T},-}}{\sqrt{\epsilon_{r}}}\right) \\
& \leq E_{r}^{\mathrm{MAX}}
\end{aligned}
$$

The inclusion of these last two constraints ensures that the hydraulic constraints of the PHES are respected when the reserves are activated. This enables PHES to provide energy arbitrage and regulation services taking into account hydraulic and power system constraints, resulting in significant cost reductions without affecting the reliability of the power system. 


\section{Reserves and PHES in IIUC}

As an alternative to the reserve requirements in the DUC, four additional ramping scenarios are introduced (set $\left.S^{\mathrm{R}}\right)$ in addition to the forecasted scenario in the IIUC [30]. As explained in [30], these ramping scenarios reduce the conservatism of the IUC solution by relaxing unnecessarily conservative inter-hour ramping requirements, as illustrated in Figure 1. In line with [30], which argues that the required interhour 'rampable capacity should be no more than the maximum up and down ramps observed over all scenarios' used in the SUC model, in this work the ramp scenarios are obtained by calculating the maximum and minimum difference between the wind power output in two adjacent time steps over all scenarios. Although the slope of these ramping scenarios is lower than in the original IUC formulation, these scenarios ensure the same capacity requirements by reaching the upper and lower bounds. We define the following four ramping scenarios:

- $s_{\mathrm{e}}^{\mathrm{R}+}$ : the maximum upward ramp of a forecast error ending on even time steps;

- $s_{\mathrm{o}}^{\mathrm{R}+}$ : the maximum upward ramp of a forecast error ending on odd time steps;

- $s_{\mathrm{e}}^{\mathrm{R}-}$ : the maximum downward ramp of a forecast error ending on even time steps;

- $s_{\mathrm{O}}^{\mathrm{R}-}$ : the maximum downward ramp of a forecast error ending on odd time steps.

A feasible dispatch enforced in each of these scenarios replaces the reserve requirements. Note that the upward and downward ramping scenarios are decoupled in two scenarios for odd and even operating intervals [30]. This decoupling is used to ensure the mathematical feasibility of the IIUC model. Without this decoupling, there would be two operating points at each time period, which would be infeasible. These ramping scenarios are however assigned a zero probability and the operational cost is calculated only for the central forecast scenario. Load shedding is not allowed in the central forecast scenario, or in the ramping scenarios. If this optimization does not yield a feasible UC schedule, market clearing condition can be relaxed for the ramping scenarios by using slack variables penalized in the objective function, as explained in [30]. In our case study, presented in Section IV, such relaxations are omitted, as all instances of the IIUC problem yielded a feasible solution.

First, we relate the energy content of the PHES in the ramping scenarios to the energy content of the PHES under forecast conditions (scenario $s^{\mathrm{F}}$ ). The energy content of the PHES is thus given by

$$
\begin{aligned}
\forall r, \forall j, \forall s \in S^{\mathrm{R}}: e_{r, j, s}= & T P \cdot\left(g_{r, j, s}^{\mathrm{PHS}, \mathrm{P}} \cdot \sqrt{\epsilon_{r}}-\frac{g_{r, j, s}^{\mathrm{PHS}, \mathrm{T}}}{\sqrt{\epsilon_{r}}}\right) \\
& +e_{r, j-1, s^{\mathrm{F}}}
\end{aligned}
$$

By linking the energy storage level in the forecast and ramping scenarios, anticipated changes in the energy storage levels are considered during the scheduling of the regulating services from the PHES. Neglecting to do so may lead to a severe underestimate of the regulating potential of the PHES. Consider for example the situation where the PHES is empty at the

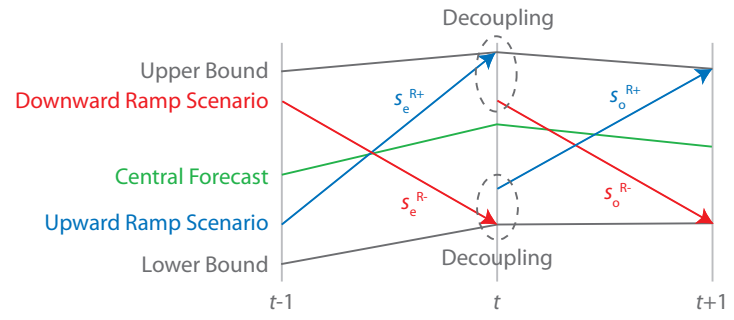

Fig. 1. Methodological illustration of the scenarios used in the IIUC [30].

start of the optimization period. Without Eq. (16), the PHES would not be allowed to offer upward regulating services by increasing its output, regardless of the evolution of the anticipated energy storage level. This equation replaces Eq. (3) for all ramping scenarios. Eq. (3) remains enforced in the forecast scenario.

Second, the output of the PHES is forced to zero at the beginning of a ramp. As the considered ramping scenarios are not real, possible realizations of the forecast error and contain non-physical transitions between time steps:

$\forall r, \forall j \mid \bmod (j)=1, \forall s \in\left\{s_{\mathrm{e}}^{\mathrm{R}+}, s_{\mathrm{e}}^{\mathrm{R}-}\right\}: g_{r, j, s}^{\mathrm{PHS}, \mathrm{P}}, g_{r, j, s}^{\mathrm{PHS}, \mathrm{T}}=0$

$\forall r, \forall j \mid \bmod (j)=0, \forall s \in\left\{s_{\mathrm{O}}^{\mathrm{R}+}, s_{\mathrm{O}}^{\mathrm{R}-}\right\}: g_{r, j, s}^{\mathrm{PHS}, \mathrm{P}}, g_{r, j, s}^{\mathrm{PHS}, \mathrm{T}}=0$

Moreover, without these constraints the non-zero PHES-based reserves at the start of a ramp may provide arbitrage opportunities, reducing the effective ramp enforced by the ramping scenario. For example, if a ramping event starts with a positive forecast error and ends with a negative forecast error, the PHES might absorb the positive forecast error at the beginning and release the stored energy at the end of the ramp, providing upward reserve. The latter action effectively reduces the ramp enforced in the IIUC and might lead to an underestimation of the flexibility needed, as the effective ramp no longer reflects the worst-case realization of the forecast error.

Third, we enforce the feasibility of the output of the PHES in the worst-case realization of the uncertain wind power (i.e., activation of all reserves in one direction):

$$
\begin{aligned}
& \forall r, \forall j, \forall s \in\left\{s_{\mathrm{e}}^{\mathrm{R}-}, s_{\mathrm{O}}^{\mathrm{R}-}\right\}: e_{r, j, s^{\mathrm{F}}}-\sum_{j^{*}=1}^{j} \sum_{s} T P \cdot \frac{\Delta^{-} g_{r, j, s}^{\mathrm{PHS}, \mathrm{T}}}{\sqrt{\epsilon_{r}}} \\
& \quad-\sum_{j^{*}=1}^{j} \sum_{s} T P \cdot \Delta^{-} g_{r, j, s}^{\mathrm{PHS}, \mathrm{P}} \cdot \sqrt{\epsilon_{r}} \geq E_{r}^{\mathrm{MIN}} \\
& \forall r, \forall j, \forall s \in\left\{s_{\mathrm{e}}^{\mathrm{R}+}, s_{\mathrm{O}}^{\mathrm{R}+}\right\}: e_{r, j, s^{\mathrm{F}}}+\sum_{j^{*}=1}^{j} \sum_{s} T P \cdot \frac{\Delta^{+} g_{r, j, s}^{\mathrm{PHS}, \mathrm{T}}}{\sqrt{\epsilon_{r}}} \\
& \quad+\sum_{j^{*}=1}^{j} \sum_{s} T P \cdot \Delta^{+} g_{r, j, s}^{\mathrm{PHS}, \mathrm{P}} \cdot \sqrt{\epsilon_{r}} \leq E_{r}^{\mathrm{MAX}}
\end{aligned}
$$

$\Delta^{-} g_{r, j, s}^{\mathrm{PHS}, \mathrm{T}}$ represents the additional output of the PHES above the output under forecast conditions. Similarly, $\Delta^{+} g_{r, j, s}^{\mathrm{PHS}, \mathrm{P}}$ represents the additional pumping power. $\Delta^{-} g_{r, j, s}^{\mathrm{PHS}, \mathrm{P}}$ and $\Delta^{+} g_{r, j, s}^{\mathrm{PHS}, \mathrm{T}}$ are corrections to the output, thus the energy content of the PHES, if it is scheduled with a non-negative output or input at that time step in both the forecast scenario 
and the ramping scenario, but in opposite directions. For example, if the PHES is scheduled to generate electricity in the forecast scenario $s^{\mathrm{F}}$, but to pump in a ramping scenario, the energy content of the PHES under worst-case conditions is affected by (i) the increase in pumping power $\left(\Delta^{+} g_{r, j, s}^{\mathrm{PHS}, \mathrm{P}}\right.$ in Eq. (20)) and (ii) the loss of output $\left(\Delta^{+} g_{r, j, s}^{\mathrm{PHS}, \mathrm{T}}\right.$ in Eq. (20)). As such, we account for the evolution of the energy content of the PHES under forecast conditions and the impact of the activation of scheduled PHES reserves on that energy content.

\section{CASE STUDY}

The computational and cost performance of the modified DUC and IIUC formulations are compared on a model of the Belgian power system, which includes 85 buses and 105 transmission lines. We assume a $30 \%$ wind energy penetration on an annual, energy basis. The $14 \mathrm{GW}$ peak demand occurs in winter, while the lowest demand - around $6 \mathrm{GW}$ - occurs during daytime in summer. The annual consumption is $83 \mathrm{TWh}$. Electrical energy generated from RES other than wind (7\% annually) is treated as a demand correction and cannot be curtailed. The demand profile and wind power data were obtained from Elia, the Belgian TSO. The conventional generation fleet consists of 71 power plants and combinedheat-and-power plants, with a total of $13,920 \mathrm{MW}$ of dispatchable capacity [32]. The nominal efficiency of each power plant is based on its type, fuel and age. The other technical characteristics of the power plants are based on ENTSO-E data [32]. One pumped hydro storage power plant has been included, with a maximum capacity of $1,308 \mathrm{MW}$, a round trip efficiency of $75 \%$ and a storage capacity of 3,924 MWh. The minimum energy content of the storage facility is set to $10 \%$ of its capacity. Since we take a system perspective, the energy storage facility is operated at no explicit cost to the system operator. However, charging/discharging of the energy storage facility incurs energy losses due to round-trip efficiencies $\epsilon_{r}<1$. These losses are taken into account when the least-cost day-ahead UC schedule is determined. The maintenance costs of all generation and transmission assets, including the energy storage facility, are neglected. The $\mathrm{CO}_{2}$-price is assumed to be $10 € /$ ton $\mathrm{CO}_{2}$. The value of lost load is $10,000 € / \mathrm{MWh}$. Curtailment of wind generation is not penalized.

The planning horizon considered in the optimization is 24 hours and the time step is 15 minutes. To ensure continuity, each optimization takes into account the values of the optimization variables over the previous 24 hours, based on the dispatch taking into account the scenario that represents the scaled measured wind power output of the previous day. Similarly, the next day is taken into account to ensure logical UC decisions and a correct evaluation of the value of stored energy in the PHES at the end of the planning horizon (24 h). To ensure a fair comparison between the different UC models, each model starts from the same set of scenarios that describes the uncertain wind power forecast. For the DUC, we impose reserve constraints equal to the maximum and minimum forecast error at each time step, as observed in the initial scenario set for each day. From the same scenario set, the ramping scenarios for the IIUC are constructed.

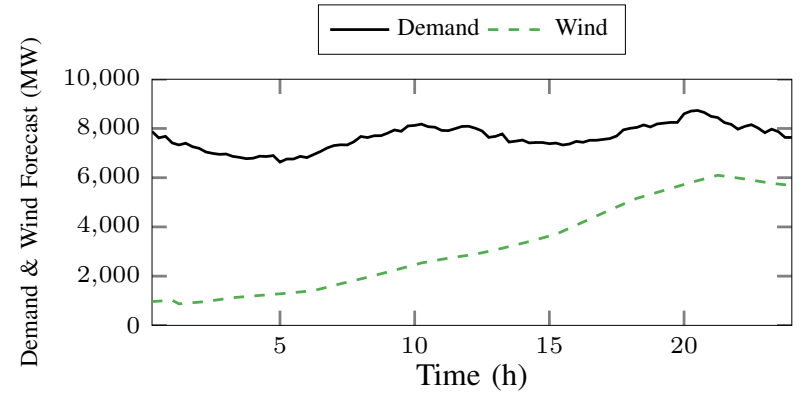

Fig. 2. The demand and forecasted wind power on the first day of week 39 . On this day, the forecasted wind output ramps up from 1,000 MW to 6,000 MW, capable of covering approx. $47 \%$ of the demand.

Note that in the DUC, probabilistic methods might be used to improve the performance of the reserve constraints [6]. Using a modified probability-distance based scenario reduction method discussed in [15], a limited set of 50 scenarios for consideration in the SUC model is selected from the same set. As such, all UC models use the same information about the wind power forecast. Differences in performance (operational system cost, wind power curtailment and reliability) are thus solely due to differences between the UC methods.

The dispatch simulations to evaluate the performance of the UC models are performed on a new set of scenarios. The number of scenarios used for the evaluation of each day-ahead schedule $\left(N^{\mathrm{MC}}\right)$ is calculated as $N^{\mathrm{MC}}=\max [500, N]$, where $N$ is the minimum number of scenarios calculated using the minimum variance method [35] for a $1 \%$ error and a $95 \%$ confidence level, as recommended in [30]. Note that $N$ is different for each day-ahead schedule, but always less than 500. This provides a proxy of the expected performance in terms of reliability and operational system cost - of the calculated UC schedule. The scenarios for these Monte Carlo simulations are generated as in [15], [32].

The model is implemented in GAMS 24.4 and MATLAB 2011b. CPLEX 12.6 is used as solver. Calculations are run on the ThinKing HPC cluster of the KU Leuven, using a $2.8 \mathrm{GHz}$ machine with 20 cores and 64GB of RAM. The duality gap was set to $0.5 \%$.

\section{REsults \& Discussion}

In Section IV-A we discuss the behavior of the DUC and IIUC models in detail, based on the simulations for a specific day. For this purpose we isolate the first day of week 39 of the calendar year, which is characterized by the systemwide demand and wind generation profiles illustrated in Fig. 2. We assume that the system operator is responsible for all scheduling decisions and transactions. Throughout this discussion we focus on the provision of the upward reserves and illustrate the importance of constraints (14)-(15) for the DUC and (16)-(20) for the IIUC. In Section IV-B we demonstrate the operational benefits of the PHES in providing regulation reserve throughout the calendar year. Before discussing the computational performance of the DUC and IIUC formulations (Section IV-D), we analyze the impact of the availability of non-spinning reserves in Section IV-C. 


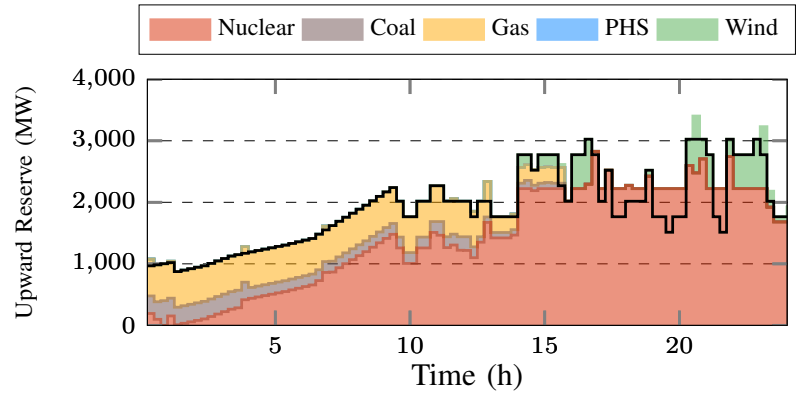

(a) PHES is not allowed to offer regulating services.

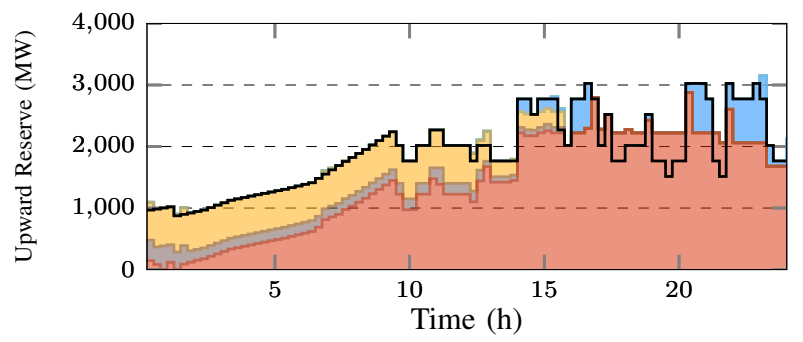

(b) PHES is allowed to offer regulating services, taking into account constraints Eq. (14)-(15).

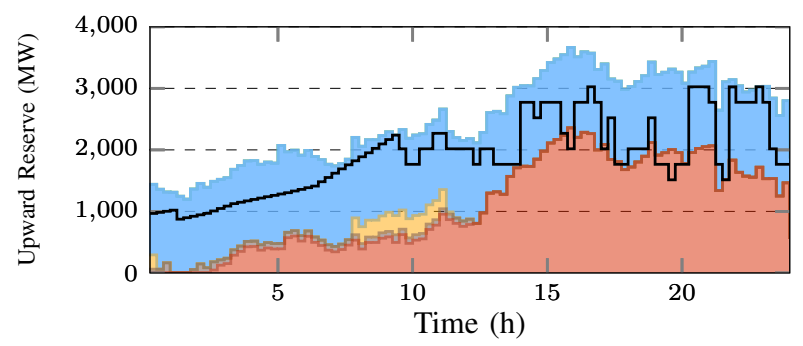

(c) PHES is allowed to offer regulating services, not taking into account constraints Eq. (14)-(15).

Fig. 3. The scheduled upward flexibility (MW) as obtained using the DUC on the first day of week 39 . The solid black line indicates the demand for upward reserves, equal to the difference between the lower bound and the central wind power forecast (Fig. 1).

\section{A. The Need for Additional Constraints on Regulating Ser- vices Offered by PHES}

Fig. 3 shows the scheduled upward reserves as obtained with the DUC model. During the first hours of the day, conventional power plants and RES curtailment are scheduled as upward regulating services if the PHES is not allowed to offer regulating services $\left(r_{r, j}^{\mathrm{PHS}, \mathrm{P},+}, r_{r, j}^{\mathrm{PHS}, \mathrm{P},-}, r_{r, j}^{\mathrm{PHS}, \mathrm{T},-}, r_{r, j}^{\mathrm{PHS}, \mathrm{T},+}=0\right)$, as shown in Fig. 3a. As the forecasted RES-based generation increases during the day, the high expected wind power results in high reserve requirements provided mainly by cheap nuclear units. The availability of the PHES to provide regulating service, constrained by Eq. (14)-(15), allows replacing mainly RES-based reserves (curtailment) by PHES-based reserves (Fig. 3b). The offered reserve power is based on the option to reduce the scheduled pumping power of the PHES. This leads to a decrease of $40 \mathrm{MW}$ in committed capacity on average, with peaks up to $433 \mathrm{MW}$. As a result, the expected operational cost decreases by $1 \%$. The expected curtailment decreases by $1422 \mathrm{MWh}$ or $42 \%$. The reliability of the resulting UC schedule is unaffected if constraints Eq. (14)-(15) are enforced. If one omits constraints (14)-(15), the DUC schedules upward

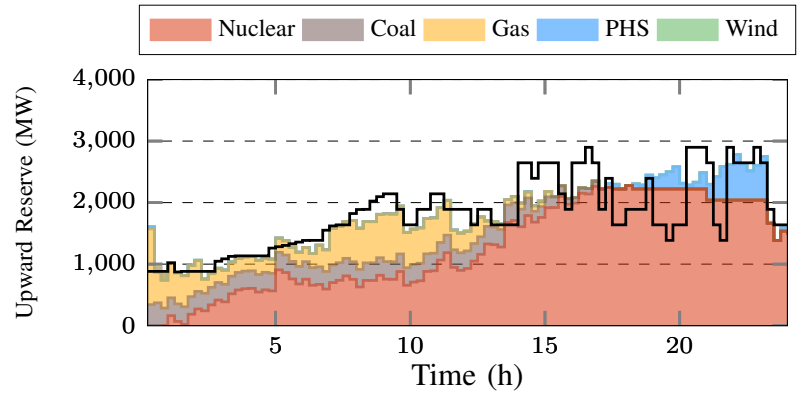

(a) PHES is not allowed to offer regulating services.

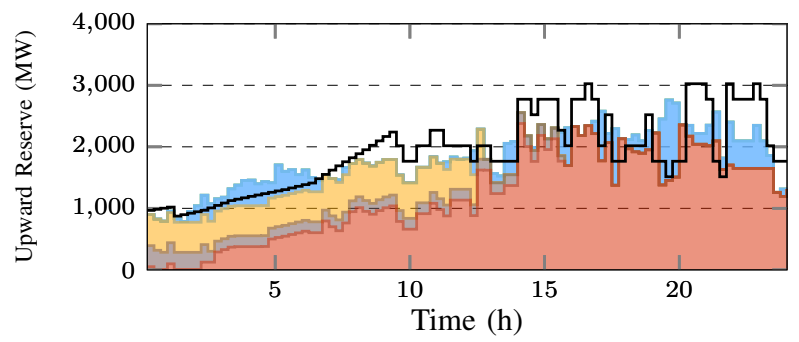

(b) PHES is allowed to offer regulating services, taking into account constraints Eq. (16)-(20).

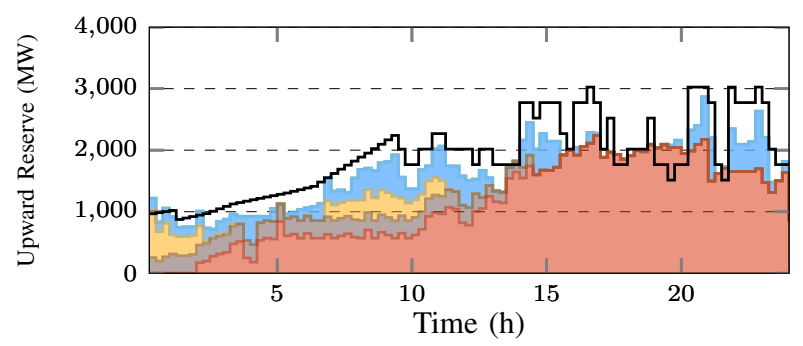

(c) PHES is allowed to offer regulating services, not taking into account constraints Eq. (16)-(20).

Fig. 4. The scheduled upward flexibility (MW) as obtained using the IIUC on the first day of week 39. The solid black line indicates the difference between the lower bound and the central wind power forecast (Fig. 1).

reserves as shown in Fig. 3c, where the PHES is continuously scheduled as upward reserve. The operational cost under forecast conditions (i.e. the operational cost as obtained from the DUC) decreases by $13 \%$, as less capacity needs to be committed to meet the demand and the reserve requirements. However, the limited energy storage capacity of PHES does not allow the activation of these reserves, resulting in load shedding during dispatch. For this specific day, the expected ENS volume reaches $354 \mathrm{MWh}$, which is to be compared with no ENS when the constraints (14)-(15) are included in the model. The expected operational costs quadruple if one accounts for the cost of ENS.

A similar analysis is shown for the IIUC in Fig. 4. As reserves are not explicitly scheduled in the IIUC, we here show the difference in output of the PHES in the downward ramping scenarios $s_{\mathrm{e}}^{\mathrm{R}-}$ and $s_{\mathrm{O}}^{\mathrm{R}-}$ compared to their output under forecast conditions (scenario $s^{\mathrm{F}}$ ). For conventional power plants, we show the available headroom of the online units, corrected for their ramping limits. When PHES is not allowed to actively participate in the implicit reserve requirements imposed by the ramping scenarios, the output of the PHES is forced to zero in the ramping scenarios $\left(\forall r, \forall j, \forall s \in S^{\mathrm{R}}\right.$ : 
$g_{r, j, s}^{\mathrm{PHS}, \mathrm{P}}, g_{r, j, s}^{\mathrm{PHS}, \mathrm{T}}=0$ ). The resulting upward reserves are illustrated in Fig. 4a. Note that the PHES implicitly 'offers' some upward reserve at the end of this day. This is in fact scheduled pumping in the central forecast scenario, which is forced to zero in the ramping scenarios. The total load in the system thus decreases in the ramping scenarios compared to the forecast scenario, which is equivalent to the activation of upward reserves. If the PHES can be used to meet the demand in the ramping scenarios, constrained by Eq. (16)-(20), the resulting upward reserves are shown in Fig. 4b. The PHES displaces some nuclear, coal- and gas-fired reserve capacity. The expected operational costs decrease by $0.5 \%$. Expected curtailment drops by $36 \%$ or $919 \mathrm{MWh}$, while the expected ENS volume is unaffected. If the constraints (16)-(20) are excluded from the model, the PHES is scheduled to provide upward reserve throughout the day (Fig. 4c). The effect is less dramatic than in the DUC (Fig. 3c), as the output of the PHES is still constrained by Eq. (3). The resulting schedule is however suboptimal: an increase in load shedding (14 MWh) fully offsets the expected operational cost reduction by actively scheduling the PHES as cost-effective upward flexibility.

In conclusion, the analysis above shows that the inclusion of the additional constraints (14)-(15) and (16)-(20) on the PHES is necessary to exploit its cost-effective regulating services in DUC and IIUC formulations. Neglecting these constraints may lead a modeler to believe the system is scheduled in a costeffective manner, while insufficient availability of flexibility will trigger load shedding during real-time operation.

\section{B. Operational Benefits of PHES Regulating Services}

Table I compares the results of the Monte-Carlo simulations for the IIUC, DUC and SUC formulations applied to the Belgian power system. These simulations include two dispatch strategies: i) the PHES provides energy arbitrage only ('Arb.'), i.e. it is used to accommodate temporary surpluses or deficits in energy, and ii) the PHES provides both energy arbitrage and regulation services ('Arb. + Reg.'). Four representative weeks were selected based on the residual demand, i.e. the demand minus the wind power generation. The week with the residual demand closest to the average weekly demand for electrical energy (week 30), the week with the lowest residual demand (week 52), the week with the highest residual energy demand (week 9) and the week with the highest variability (week 39) were selected. In the DUC and IIUC, the additional constraints proposed in Section II are enforced when the PHES unit is allowed to provide regulating services.

A number of trends can be identified in the results summarized in Table I. First, allowing scheduling of regulating services offered by the PHES reduces the expected operational cost at the expense of a decreased reliability. Indeed, for all weeks except and both the DUC and IIUC, the operational cost decreases significantly. Especially at high shares of wind energy (week 39, high upward reserve requirements and flexible, expensive units needed to meet this requirement) and in weeks characterized by a high residual demand (week 9, low reserve requirements, but only expensive units available due to a high demand) the decrease in operational cost is significant. Operational cost savings vary between 0 and 1.2
$\mathrm{M} € /$ week. This cost reduction is the result of i) an improved utilization of the available wind power and ii) a more costefficient UC schedule. The first effect is illustrated by the Wind Utilization Factor (WUF) in Table I, and the second was discussed in Section IV-A. By enforcing Eq. (14)-(15) and (16)-(20), the reliability of the UC schedules is unaffected by the presence of PHES-based reserves. ENS accounts at most for $0.004 \mathrm{M} € /$ week of the total operational cost. The ENS volumes represent less than $0.0001 \%$ of the load in all cases.

Second, the DUC consistently outperforms the IIUC if the PHES unit is used only for energy arbitrage. Although both models are constrained by the same reserve capacity requirements (upper and lower bound in Fig. 1), RES-based reserves are treated differently. In the DUC, curtailed forecasted RES-based generation may be used to meet the demand for upward reserves, which the IIUC does not allow. The IIUC therefore must schedule more capacity to meet the upward reserve requirements, which leads to higher operational costs. PHES-based regulating services allow to meet the reserve requirements with less capacity, leading to a higher WUF for the DUC and IIUC solutions. Operational costs with PHESbased regulating services are similar for both the DUC and IIUC, with lower expected operational costs for the IIUC solutions in week 39 and 52.

Third, one can compare these results to those obtained with the SUC model. We imposed a time limit of 50,000 seconds on the optimization. In some cases, this was insufficient to find a solution of the SUC problem that satisfies the duality gap. For week 30, 3 out of 7 SUC optimization problems did not yield a solution that satisfies the $0.5 \%$ dual gap within the time limit. The median value of the resulting dual gaps is $0.95 \%$. In week 39,3 suboptimal solutions out of 7 yielded a median dual gap of $0.49 \%$. One UC problem was not solved to optimality in week 9, which resulted in a median dual gap of $0.31 \%$. Two UC schedules did not satisfy the $0.5 \%$ dual gap in week 52, which results in a median dual gap of $0.95 \%$. Note that these values should not be interpreted as a relative cost reduction that could still be possible because they might be the result of a lack of convergence of the lower bound. For the days whose found SUC solutions that (almost) satisfy the duality gap, the SUC outperforms the IIUC and DUC. In week 9, the SUC outperforms the DUC and IIUC by $2.1 \%$ and $3.9 \%$, respectively, when the PHES is only used for arbitrage, and $1.4 \%$ if the PHES offers regulating services as well. However, due to the presence of some suboptimal solutions in weeks 30 and 52, the SUC is outperformed by the IIUC and DUC. For example, in week 52, the SUC yields operational cost reductions up to $69 \%$ per day in three out of seven days compared to the IIUC (30\% overall). However, due to the presence of four suboptimal solutions, the global performance of the SUC is 5.7 to $11.4 \%$ worse than that of the IIUC. Furthermore, the SUC typically results in more load shedding. This is partly the result of the presence of suboptimal solutions, but also a consequence of actively scheduling load shedding in unlikely, extreme scenarios, which is not possible in the IIUC and DUC. This load shedding represents a significant part of the total operational cost, but the curtailed load is at most $0.006 \%$ of the total demand. 
TABLE I

COMPARISON OF THE DUC, IIUC AND SUC DURING FOUR REPRESENTATIVE WEEKS. ALL VALUES ARE GIVEN PER WEEK. NON-SPINNING RESERVES ARE NOT CONSIDERED. TC IS THE TOTAL OPERATIONAL COST AND ENS THE ENERGY NOT SUPPLIED VOLUME.

WUF IS THE WIND UTILIZATION FACTOR, THE PERCENTAGE OF AVAILABLE WIND POWER THAT IS ABSORBED IN THE POWER SYSTEM. SW IS THE SHARE OF WIND POWER IN THE TOTAL DEMAND FOR ELECTRICAL ENERGY.

\begin{tabular}{c|cc|cc|c}
\hline \hline & \multicolumn{2}{|c|}{ DUC } & \multicolumn{2}{c|}{ IIUC } & SUC \\
& Arb. & Arb. + Reg. & Arb. & Arb. + Reg. & Arb. + Reg. \\
\hline \hline TC (M€) & 13.8 & 13.8 & 14.2 & 13.8 & 14.2 \\
ENS (MWh) & 0 & 0 & 0 & 0.4 & 41.6 \\
WUF (\%) & 100 & 100 & 100 & 100 & 100 \\
SW (\%) & 10.6 & 10.6 & 10.6 & 10.6 & 10.6 \\
\hline \hline
\end{tabular}

(a) Week 30 (average residual demand)

\begin{tabular}{c|cc|cc|c}
\hline \hline & \multicolumn{2}{|c|}{ DUC } & \multicolumn{2}{c|}{ IIUC } & SUC \\
& Arb. & Arb. + Reg. & Arb. & Arb. + Reg. & Arb. + Reg. \\
\hline \hline TC (M€) & 28.7 & 28.5 & 29.2 & 28.5 & 28.1 \\
ENS (MWh) & 0 & 0 & 0 & 0 & 16.0 \\
WUF (\%) & 100 & 100 & 100 & 100 & 100 \\
SW (\%) & 13.5 & 13.5 & 13.5 & 13.5 & 13.5 \\
\hline \hline
\end{tabular}

(b) Week 9 (highest residual demand)

\begin{tabular}{c|cc|cc|c}
\hline \hline & \multicolumn{2}{|c|}{ DUC } & \multicolumn{2}{c|}{ IIUC } & SUC \\
& Arb. & Arb. + Reg. & Arb. & Arb. + Reg. & Arb. + Reg. \\
\hline \hline TC (M€) & 3.3 & 3.3 & 3.5 & 3.1 & 3.5 \\
ENS (MWh) & 0 & 0 & 0 & 0 & 85.0 \\
WUF (\%) & 85.8 & 86.7 & 83.8 & 86.5 & 91.0 \\
SW (\%) & 74.0 & 74.8 & 72.2 & 74.6 & 79.0 \\
\hline
\end{tabular}

(c) Week 52 (lowest residual demand)

\begin{tabular}{c|cc|cc|c}
\hline \hline & \multicolumn{2}{|c|}{ DUC } & \multicolumn{2}{c|}{ IIUC } & SUC \\
& Arb. & Arb. + Reg. & Arb. & Arb. + Reg. & Arb. + Reg. \\
\hline \hline TC (M€) & 9.0 & 8.8 & 9.8 & 8.6 & 8.4 \\
ENS (MWh) & 0 & 0 & 0 & 0 & 94.3 \\
WUF (\%) & 93.5 & 93.5 & 92.3 & 93.2 & 96.0 \\
SW (\%) & 48.9 & 48.9 & 48.3 & 48.8 & 50.2 \\
\hline \hline
\end{tabular}

(d) Week 39 (most variable residual demand)

\section{Impact of the Non-Spinning Reserves on the Value of PHES-based Regulating Services}

As non-spinning reserves can reduce the cost of regulating services, their presence will affect the value of the PHESbased regulating services. To analyze this effect, we allow highly flexible power plants to be scheduled as non-spinning reserve. These highly flexible generators include open-cycle gas- and oil-fired power plants with a capacity of less than 100 MW, minimum up- and down times of 1 time step and the capability to ramp from zero output to full capacity within 1 time step. In total, 35 fast-starting units with a total capacity of 1,118 MW are considered in this case study. In the DUC and IIUC, these units can be scheduled at zero cost to meet the reserve requirements and the demand in the ramping scenarios respectively. In the SUC, non-spinning reserves are explicitly scheduled by allowing a scenario-specific unit commitment status for fast-starting units. The cost of activating nonspinning reserve is accounted for in the objective function of the SUC model [32]. The SUC considers 40 scenarios. The
TABLE II

COMPARISON OF THE DUC, IIUC AND SUC DURING FOUR REPRESENTATIVE WEEKS. ALL VALUES ARE GIVEN PER WEEK. NON-SPINNING RESERVES ARE CONSIDERED. TC IS THE TOTAL OPERATIONAL COST AND ENS THE ENERGY NOT SUPPLIED VOLUME.

WUF IS THE WIND UTILIZATION FACTOR, THE PERCENTAGE OF AVAILABLE WIND POWER THAT IS ABSORBED IN THE POWER SYSTEM. SW IS THE SHARE OF WIND POWER IN THE TOTAL DEMAND FOR ELECTRICAL ENERGY.

\begin{tabular}{c|cc|cc|c}
\hline \hline & \multicolumn{2}{|c|}{ DUC } & \multicolumn{2}{c|}{ IIUC } & SUC \\
& Arb. & Arb. + Reg. & Arb. & Arb. + Reg. & Arb. + Reg. \\
\hline \hline TC (M€) & 13.2 & 13.2 & 13.2 & 13.2 & 13.4 \\
ENS (MWh) & 0.5 & 0.7 & 0.8 & 0.5 & 22.5 \\
WUF (\%) & 100 & 100 & 100 & 100 & 100 \\
SW (\%) & 10.6 & 10.6 & 10.6 & 10.6 & 10.6 \\
\hline \hline
\end{tabular}

(a) Week 30 (average residual demand)

\begin{tabular}{c|cc|cc|c}
\hline \hline & \multicolumn{2}{|c|}{ DUC } & \multicolumn{2}{c|}{ IIUC } & SUC \\
& Arb. & Arb. + Reg. & Arb. & Arb. + Reg. & Arb. + Reg. \\
\hline \hline TC (M€) & 27.5 & 27.5 & 27.7 & 27.5 & 27.2 \\
ENS (MWh) & 2.0 & 1.8 & 1.7 & 4.5 & 8.1 \\
WUF (\%) & 100 & 100 & 100 & 100 & 100 \\
SW (\%) & 13.5 & 13.5 & 13.5 & 13.5 & 13.5 \\
\hline \hline
\end{tabular}

(b) Week 9 (highest residual demand)

\begin{tabular}{c|cc|cc|c}
\hline \hline & \multicolumn{2}{|c|}{ DUC } & \multicolumn{2}{c|}{ IIUC } & SUC \\
& Arb. & Arb. + Reg. & Arb. & Arb. + Reg. & Arb. + Reg. \\
\hline \hline TC (M€) & 2.4 & 2.4 & 2.7 & 2.5 & 2.9 \\
ENS (MWh) & 0.2 & 0.1 & 0 & 0.5 & 65.5 \\
WUF (\%) & 91.5 & 92.4 & 88.7 & 91.9 & 94.1 \\
SW (\%) & 79.0 & 79.7 & 76.5 & 79.2 & 81.1 \\
\hline \hline
\end{tabular}

(c) Week 52 (lowest residual demand)

\begin{tabular}{|c|c|c|c|c|c|}
\hline & Arb. & $\begin{array}{l}\text { DUC } \\
\text { Arb. + Reg. }\end{array}$ & Arb. & $\begin{array}{l}\text { IIUC } \\
\text { Arb. + Reg. }\end{array}$ & $\begin{array}{c}\text { SUC } \\
\text { Arb. + Reg. }\end{array}$ \\
\hline $\mathrm{TC}(\mathrm{M} €)$ & 7.1 & 6.9 & 7.7 & 6.9 & 6.3 \\
\hline ENS (MWh) & 0.6 & 0.2 & 0.2 & 0.7 & 10.7 \\
\hline WUF (\%) & 95.8 & 96.0 & 94.8 & 95.6 & 97.2 \\
\hline SW (\%) & 50.1 & 50.3 & 49.6 & 50.0 & 50.9 \\
\hline
\end{tabular}

(d) Week 39 (most variable residual demand)

results shown are an updated version of those published in [32]. The duality gap is reached for all instances.

Scheduling of non-spinning reserve reduces the operating cost by $0.6 \mathrm{M} € /$ week to $2.1 \mathrm{M} € /$ week (3.7 to $37.5 \%$, respectively - see Table II). These reductions are especially noticeable during the weeks with high RES-based electricity generation, e.g. weeks 39 and 52, where they result in cost savings of $22 \%$ to $37 \%$ and $21 \%$ to $27 \%$, respectively. This is the result of an increased wind power utilization (up to $7 \%$ ), which replaces conventional generation and, thus, meets the balancing needs at a lower expected activation cost of non-spinning reserve. On the other hand, non-spinning reserve reduces the value of regulating services provided by the PHES unit. Thus, the PHES unit results in cost savings up to 0.8 $\mathrm{M} € /$ week (week 39), if non-spinning reserve is scheduled, as compared to $1.2 \mathrm{M} € /$ week (week 39) of cost savings, if non-spinning reserve is not scheduled. Furthermore, in some cases, such as week 30 and 9, the PHES unit does not provide any cost savings, because non-spinning reserves can provide regulating service more cost-effectively. 
TABLE III

COMPARISON OF THE CPU TIME (S) PER RUN OF THE DUC, IIUC AND SUC. P(75) IS THE $75^{\text {th }}$ PERCENTILE.

\begin{tabular}{c|cc|cc|c}
\hline \hline & \multicolumn{2}{|c|}{ DUC } & \multicolumn{2}{c|}{ IIUC } & SUC \\
& Arb. & Arb. + Reg. & Arb. & Arb. + Reg. & Arb. + Reg. \\
\hline \hline Median & 67 & 138 & 228 & 244 & $+50,000$ \\
P(75) & 93 & 191 & 507 & 328 & $+50,000$ \\
P(90) & 241 & 342 & 768 & 579 & $+50,000$ \\
\hline \hline
\end{tabular}

(a) Without non-spinning reserves

\begin{tabular}{c|cc|cc|c}
\hline \hline & \multicolumn{2}{|c|}{ DUC } & \multicolumn{2}{c|}{ IIUC } & SUC \\
& Arb. & Arb. + Reg. & Arb. & Arb. + Reg. & Arb. + Reg. \\
\hline \hline Median & 61 & 125 & 217 & 177 & 28,921 \\
P(75) & 91 & 213 & 400 & 289 & 36,296 \\
P(90) & 188 & 335 & 8,345 & 553 & $+50,000$ \\
\hline \hline
\end{tabular}

(b) With non-spinning reserves

Compared to the solutions of a SUC, considering nonspinning reserves, the operational cost obtained with a DUC or IIUC remains high in week 9 and 39. In weeks 30 and 52, the differences between the results of the considered UC models are less pronounced. In both weeks, the limited scenario set considered during UC does not contain sufficient information to avoid load shedding during dispatch. As a result, the operational cost is higher for the UC obtained with the SUC model. If the cost of load shedding is excluded, the SUC yields an expected operational cost of 2.3 $\mathrm{M} €$ (week 52) and 13.2 $\mathrm{M} €$ (week 30) respectively.

\section{Computational Performance}

The resulting execution times (median and percentiles) for all UC models are shown in Table III. The DUC typically solves the quickest, followed by the IIUC. In general, the DUC takes more time to solve if the PHES is allowed to offer regulating services. Solving a DUC takes approximately one to two minutes. If the PHES is not allowed to offer regulating services in the IIUC, the median calculation time is approximately 3.8 minutes. If PHES provides regulating services, calculation times rise to 4.1 minutes (median). Furthermore, note that the CPU times of the IIUC show a much larger spread on the calculation time than those of the DUC. Compared to the SUC, the IIUC is still considerably less computationally challenging. We also noted that the SUC fails to yield a solution that satisfies the duality gap of $0.5 \%$ in 50,000 seconds in over $50 \%$ of the cases.

The presence of non-spinning reserves lowers the computational cost of the SUC considerably, despite the presence of more binary variables. The computational performance of the DUC is unchanged. The computational cost of the IIUC decreases considerably if non-spinning and PHES-based reserves are available (median values).

\section{CONCLUSION}

The stochastic nature of renewable electricity generation requires system operators to schedule controllable generation in such a way that sufficient flexibility is available in realtime to compensate for mismatches between the forecasted and realized renewable electricity generation. The costs associated with procuring and activating this flexibility should be minimized. Cost-efficient flexibility can be introduced by pumped hydro energy storage systems (PHES). However, the hydraulic constraints of these systems further increase the complexity of the scheduling problem faced by a system operator. Several frameworks exist to study this scheduling problem, such as deterministic unit commitment (DUC), (improved) interval UC ((I)IUC), robust UC (RUC) and stochastic UC (SUC). RUC and SUC approaches avoid violations of the hydraulic constraints of a PHES by dispatching the PHES in each considered scenario, but can be computationally intensive. DUC and (I)IUC models are sufficient to study energy arbitrage with PHES and are easy to solve, but fail to account for the hydraulic constraints of the PHES when offering regulating services.

In this paper, we propose novel PHES constraints and cooptimize the PHES dispatch decisions with the unit commitment decisions of the conventional power plants when offering regulating services in a DUC and IIUC model. The proposed constraints are necessary to exploit the cost-effective regulating services that a PHES may offer in a DUC or IIUC. The proposed IUC and DUC models achieve significant cost savings, increased wind power utilization and small CPU time increases. The IIUC attains lower operational costs than the DUC, especially when at high wind energy penetration levels. The IIUC allows approximating the cost-optimal solution of the SUC at a much lower computational cost.

\section{ACKNOWLEDGMENT}

The computational resources and services used in this work were provided by the Hercules Foundation and the Flemish Government - department EWI.

\section{REFERENCES}

[1] E. Ela and M. O'Malley, "Studying the Variability and Uncertainty Impacts of Variable Generation at Multiple Timescales," IEEE Transactions on Power Systems, vol. 27, no. 3, pp. 1324-1333, Aug. 2012.

[2] K. Van Den Bergh, K. Bruninx, E. Delarue, and W. D'haeseleer, "A Mixed-Integer Linear Formulation of the Unit Commitment Problem," WP EN2014-07, 2014.

[3] M. A. Ortega-Vazquez and D. S. Kirschen, "Assessing the Impact of Wind Power Generation on Operating Costs," IEEE Transactions on Smart Grid, vol. 1, no. 3, pp. 295-301, Dec. 2010.

[4] _ - "Should the spinning reserve procurement in systems with wind power generation be deterministic or probabilistic?" in 2009 International Conference on Sustainable Power Generation and Supply. IEEE, Apr. 2009, pp. 1-9.

[5] _ "Estimating the Spinning Reserve Requirements in Systems With Significant Wind Power Generation Penetration," IEEE Transactions on Power Systems, vol. 24, no. 1, pp. 114-124, Feb. 2009.

[6] K. Bruninx and E. Delarue, "A Statistical Description of the Error on Wind Power Forecasts for Probabilistic Reserve Sizing," IEEE Transactions on Sustainable Energy, vol. 5, no. 3, pp. 995-1002, Jul. 2014.

[7] Y. Dvorkin, D. S. Kirschen, and M. A. Ortega-Vazquez, "Assessing flexibility requirements in power systems," IET Generation, Transmission \& Distribution, vol. 8, no. 11, pp. 1820-1830, Nov. 2014.

[8] A. Papavasiliou, S. S. Oren, and R. P. O’Neill, "Reserve Requirements for Wind Power Integration: A Scenario-Based Stochastic Programming Framework," IEEE Transactions on Power Systems, vol. 26, no. 4, pp. 2197-2206, Nov. 2011.

[9] Y. Wang, Q. Xia, and C. Kang, "Unit Commitment With Volatile Node Injections by Using Interval Optimization," IEEE Transactions on Power Systems, vol. 26, no. 3, pp. 1705-1713, Aug. 2011. 
[10] D. Bertsimas, E. Litvinov, X. A. Sun, J. Zhao, and T. Zheng, "Adaptive Robust Optimization for the Security Constrained Unit Commitment Problem," IEEE Transactions on Power Systems, vol. 28, no. 1, pp. 52-63, Feb. 2013

[11] D. Lew, G. Brinkman, E. Ibanez, B. Hodge, and J. King, "Western wind and solar integration study," National Renewable Energy Laboratory NREL/TP-5500, Tech. Rep. December, 2010.

[12] C. Zhao and Y. Guan, "Unified Stochastic and Robust Unit Commitment," IEEE Transactions on Power Systems, vol. 28, no. 3, pp. 33533361, Aug. 2013.

[13] A. Papavasiliou, S. S. Oren, and B. Rountree, "Applying High Performance Computing to Transmission-Constrained Stochastic Unit Commitment for Renewable Energy Integration," IEEE Transactions on Power Systems, vol. 30, no. 3, pp. 1109-1120, May 2015

[14] Y. Dvorkin, Y. Wang, H. Pandzic, and D. Kirschen, "Comparison of scenario reduction techniques for the stochastic unit commitment," in 2014 IEEE PES General Meeting - Conference \& Exposition. IEEE, Jul. 2014, pp. 1-5.

[15] K. Bruninx, E. Delarue, and W. D'haeseleer, "A practical approach on scenario generation \& reduction algorithms based on probability distance measures - the case of wind power forecast errors," WP EN2014-15, 2014.

[16] P. Pinson and G. Kariniotakis, "Conditional Prediction Intervals of Wind Power Generation," in IEEE Transactions on Power Systems, vol. 25, no. 4, Nov. 2010, pp. 1845-1856.

[17] G. Yongpei and J. Wang, "Uncertainty Sets for Robust Unit Commitment," IEEE Transactions on Power Systems, vol. 29, no. 3, pp. 14391440, May 2014.

[18] D. Bertsimas and M. Sim, "The price of robustness," Operations research, vol. 52, pp. 35-53, 2004.

[19] A. Lorca and A. Sun, "Adaptive Robust Optimization with Dynamic Uncertainty Sets for Multi-Period Economic Dispatch under Significan Wind," Accepted for publication in IEEE Transactions on Power Systems, no. September, pp. 1-16, Sep. 2014.

[20] D. Bertsimas and M. Sim, "Tractable Approximations to Robust Conic Optimization Problems," Mathematical Programming, vol. 107, no. 1-2, pp. 5-36, Dec. 2005

[21] W. Lei, M. Shahidehpour, and Z. Li, "Comparison of Scenario-Based and Interval Optimization Approaches to Stochastic SCUC," IEEE Transactions on Power Systems, vol. 27, no. 2, pp. 913-921, May 2012.

[22] Y. V. Makarov, P. Du, M. C. W. Kintner-Meyer, C. Jin, and H. F. Illian, "Sizing Energy Storage to Accommodate High Penetration of Variable Energy Resources," IEEE Transactions on Sustainable Energy, vol. 3 , no. 1, pp. 34-40, Jan. 2012.

[23] D. Pozo, J. Contreras, and E. E. Sauma, "Unit Commitment With Ideal and Generic Energy Storage Units," IEEE Transactions on Power Systems, vol. 29, no. 6, pp. 2974-2984, Nov. 2014.

[24] H. Pandzic, Y. Wang, T. Qiu, Y. Dvorkin, and D. S. Kirschen, "NearOptimal Method for Siting and Sizing of Distributed Storage in a Transmission Network," IEEE Transactions on Power Systems, pp. 1-13, 2014.

[25] NHA's Pumped Storage Development Council, "Challenges and Opportunities For New Pumped Storage Development," Tech. Rep., 2012.

[26] A. Kalantari and F. D. Galiana, "On the significance of hydro storage in leveraging wind integration in thermal systems," in 20129 th International Conference on the European Energy Market. IEEE, May 2012 , pp. 1-8.

[27] R. Aihara, A. Yokoyama, F. Nomiyama, and N. Kosugi, "Optimal operation scheduling of pumped storage hydro power plant in power system with a large penetration of photovoltaic generation using genetic algorithm," in 2011 IEEE Trondheim PowerTech. IEEE, Jun. 2011, pp. $1-8$.

[28] S. J. Kazempour, M. Hosseinpour, and M. P. Moghaddam, "Selfscheduling of a joint hydro and pumped-storage plants in energy, spinning reserve and regulation markets," in 2009 IEEE Power \& Energy Society General Meeting. IEEE, Jul. 2009, pp. 1-8.

[29] R. Jiang, J. Wang, and Y. Guan, "Robust Unit Commitment With Wind Power and Pumped Storage Hydro," IEEE Transactions on Power Systems, vol. 27, no. 2, pp. 800-810, May 2012.

[30] H. Pandzic, Y. Dvorkin, T. Qui, Y. Wang, and D. S. Kirschen, "Toward Cost-Efficient and Reliable Unit Commitment Under Uncertainty," IEEE Transactions on Power Systems, vol. PP, 2015.

[31] Y. Dvorkin, H. Pandzic, M. A. Ortega-Vazquez, and D. S. Kirschen, "A Hybrid Stochastic/Interval Approach to Transmission-Constrained Unit Commitment," IEEE Transactions on Power Systems, vol. 30, no. 2, pp. 621-631, Mar. 2015.
[32] K. Bruninx, K. Van Den Bergh, E. Delarue, and W. D'haeseleer, "Optimization and allocation of spinning reserves in a low carbon framework," IEEE Transactions on Power Systems, vol. PP, pp. 1-8, 2015.

[33] E. Lannoye, D. Flynn, and M. O’Mally, "Transmission, Variable Generation, and Power System Flexibility," IEEE Transactions on Power Systems, vol. 30, no. 1, pp. 1-10, 2015.

[34] K. Van den Bergh, D. Couckuyt, E. Delarue, and W. D'haeseleer, "Redispatching in an interconnected electricity system with high renewables penetration," Electric Power System Research, vol. 127, no. 10, pp. 64 $72,2015$.

[35] G. Hahn and S. Shapiro, Statistical Models in Engineering. New York, NY, USA: Wiley, 1967.

Kenneth Bruninx (S'15) received the M.S. degree in energy engineering in 2011 from the Univeristy of Leuven (KU Leuven). Currently, he is working as a PhD-researcher at the University of Leuven Energy Institute, TME branch (energy conversion)

Yury Dvorkin (S'11) received the B.S.E.E degree with the highest honors at Moscow Power Engineering Institute (Technical University), Moscow, Russia in 2011. He is currently pursuing the Ph.D. degree in electrical engineering at the University of Washington, Seattle, WA, USA. He is a recipient of the Clear Energy Institute's Graduate Fellowship (2013-2014) and the Clean Energy Institute Student Training \& Exploration Grant (2014-2015). His research interests include short- and long-term planning in power systems with renewable generation and power system economics.

Erik Delarue (M'15) received the M.S. degree in mechanical engineering in 2005 and the Ph.D. degree in mechanical engineering in 2009, both from the University of Leuven (KU Leuven), Belgium. Currently, he is a postdoctoral research fellow of the Research Foundation - Flanders (F.W.O.), at the University of Leuven Energy Institute, TME branch (energy conversion)

Hrvoje Pandžić (S'06 -M'12) received the M.E.E. and Ph.D. degrees from the Faculty of Electrical Engineering and Computing, University of Zagreb, Croatia, in 2007 and 2011, respectively. In the period 2012-2014 he was a postdoctoral researcher at the University of Washington, Seattle, WA, USA Currently, he is an Assistant Professor at the Faculty of Electrical Engineering and Computing, University of Zagreb. His research interests include planning, operation, control and economics of power and energy systems.

William D'haeseleer received his M.S. degree in Electro-Mechanical Engineering and a Master in Nuclear Engineering from the University of Leuven (KU Leuven), Belgium, in 1980 and 1982, respectively. He obtained another M.S. degree in Electrical Engineering and the Ph.D. degree from the University of Wisconsin-Madison, USA, in 1983 and 1988, respectively. Currently, he is a full-time Professor in the College of Engineering of the KU Leuven and Director of the University of Leuven Energy Institute.

Daniel S. Kirschen (M'86-SM'91-F'07) received the electrical and mechanical engineer's degrees from the Université Libre de Bruxelles, Belgium, in 1979, and the M.Sc. and Ph.D. degrees from the University of Wisconsin, Madison, WI, USA, in 1980 and 1985, respectively. He is currently Close Professor of Electrical Engineering at the University of Washington, Seattle, WA, USA. His research focuses on smart grids, the integration of renewable energy sources in the grid, power system economics and power system security. 